G. Ottaviani

Nagoya Math. J.

Vol. 193 (2009), 95-110

\title{
AN INVARIANT REGARDING WARING'S PROBLEM FOR CUBIC POLYNOMIALS
}

\author{
GIORGIO OTTAVIANI
}

to the memory of Michael Schneider, ten years after

\begin{abstract}
We compute the equation of the 7-secant variety to the Veronese variety $\left(\mathbf{P}^{4}, \mathcal{O}(3)\right)$, its degree is 15 . This is the last missing invariant in the Alexander-Hirschowitz classification. It gives the condition to express a homogeneous cubic polynomial in 5 variables as the sum of 7 cubes (Waring problem). The interesting side in the construction is that it comes from the determinant of a matrix of order 45 with linear entries, which is a cube. The same technique allows to express the classical Aronhold invariant of plane cubics as a pfaffian.
\end{abstract}

\section{$\S 1$. Introduction}

We work over an algebraically closed field $K$ of characteristic zero. The Veronese variety, given by $\mathbf{P}^{n}$ embedded with the linear system $|\mathcal{O}(d)|$, lives in $\mathbf{P}^{N}$ where $N=\left(\begin{array}{c}n+d \\ d\end{array}\right)-1$. It parametrizes the homogeneous polynomials $f$ of degree $d$ in $n+1$ variables which are the power of a linear form $g$, that is $f=g^{d}$.

Let $\sigma_{s}\left(\mathbf{P}^{n}, \mathcal{O}(d)\right)$ be the $s$-secant variety of the Veronese variety, that is the Zariski closure of the variety of polynomials $f$ which are the sum of the powers of $s$ linear forms $g_{i}$, i.e. $f=\sum_{i=1}^{s} g_{i}^{d}$. In particular $\sigma_{1}\left(\mathbf{P}^{n}, \mathcal{O}(d)\right)=$ $\left(\mathbf{P}^{n}, \mathcal{O}(d)\right)$ is the Veronese variety itself and $\sigma_{2}\left(\mathbf{P}^{n}, \mathcal{O}(d)\right)$ is the usual secant variety. For generalities about the Waring's problem for polynomials see [IK] or [RS].

Our starting point is the theorem of Alexander and Hirschowitz (see $[\mathrm{AH}]$ or $[\mathrm{BO}]$ for a survey, including a self-contained proof) which states that the codimension of $\sigma_{s}\left(\mathbf{P}^{n}, \mathcal{O}(d)\right) \subseteq \mathbf{P}^{N}$ is the expected one, that is $\max \{N+1-(n+1) s, 0\}$, with the only exceptions

(i) $\sigma_{k}\left(\mathbf{P}^{n}, \mathcal{O}(2)\right), 2 \leq k \leq n$

Received December 18, 2007.

Accepted May 7, 2008.

2000 Mathematics Subject Classification: 15A72, 14L35, 14M12, 14M20. 
(ii) $\sigma_{\frac{1}{2} n(n+3)}\left(\mathbf{P}^{n}, \mathcal{O}(4)\right), n=2,3,4$

(iii) $\sigma_{7}\left(\mathbf{P}^{4}, \mathcal{O}(3)\right)$

The case (i) corresponds to the matrices of rank $\leq k$ in the variety of symmetric matrices of order $n+1$. In the cases (ii) and (iii) the expected codimension is zero, while the codimension is one. Hence the equation of the hypersurface $\sigma_{s}\left(\mathbf{P}^{n}, \mathcal{O}(d)\right)$ in these cases is an interesting $S L(n+$ 1)-invariant. In the cases (ii) it is the catalecticant invariant, that was computed by Clebsch in the 19th century, its degree is $\left(\begin{array}{c}n+2 \\ 2\end{array}\right)$.

The main result of this paper is the computation of the equation of $\sigma_{7}\left(\mathbf{P}^{4}, \mathcal{O}(3)\right)$. This was left as an open problem in [IK, Chap. 2, Rem. 2.4].

We consider a vector space $V$. For any nonincreasing sequence of positive integers $\alpha=\left(\alpha_{1}, \alpha_{2}, \ldots\right)$ it is defined the Schur module $\Gamma^{\alpha} V$, which is an irreducible $S L(V)$-module (see $[\mathrm{FH}])$. For $\alpha=(p)$ we get the $p$-th symmetric power of $V$ and for $\alpha=(1, \ldots, 1)$ ( $p$ times) we get the $p$-th alternating power of $V$. The module $\Gamma^{\alpha} V$ is visualized as a Young diagram containing $\alpha_{i}$ boxes in the $i$-th row. In particular if $\operatorname{dim} V=5$ then $\Gamma^{2,2,1,1} V$ and its dual $\Gamma^{2,1,1} V$ have both dimension 45 .

Our main result is the following

THEOREM 1.1. Let $V$ be a vector space of dimension 5. For any $\phi \in$ $S^{3} V$, let $B_{\phi}: \Gamma^{2,2,1,1} V \rightarrow \Gamma^{2,1,1} V$ be the $S L(V)$-invariant contraction operator. Then there is an irreducible homogeneous polynomial $P$ of degree 15 on $S^{3} V$ such that

$$
2 P(\phi)^{3}=\operatorname{det} B_{\phi}
$$

The polynomial $P$ is the equation of $\sigma_{7}(\mathbf{P}(V), \mathcal{O}(3))$.

The coefficient 2 is needed because we want the invariant polynomials to be defined over the rational numbers. The picture in terms of Young diagrams is

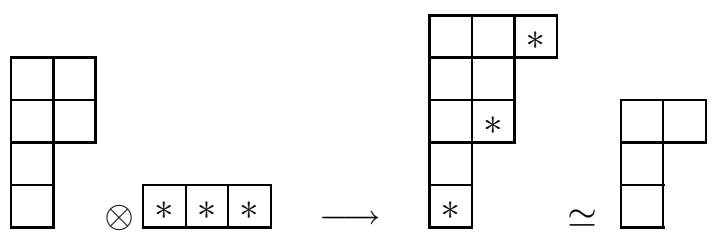

This picture means that $\Gamma^{2,1,1} V$ is a direct summand of the tensor product $\Gamma^{2,2,1,1} V \otimes S^{3} V$, according to the Littlewood-Richardson rule $([\mathrm{FH}])$. 
The polynomial $P$ gives the necessary condition to express a cubic homogeneous polynomial in five variables as a sum of seven cubes. We prove in Lemma 3.2 that if $\phi$ is decomposable then $\operatorname{rk}\left(B_{\phi}\right)=6$. The geometrical explanation that $\sigma_{7}\left(\mathbf{P}^{4}, \mathcal{O}(3)\right)$ is an exceptional case is related to the fact that given seven points in $\mathbf{P}^{4}$ there is a unique rational normal curve through them, and it was discovered independently by Richmond and Palatini in 1902, see $[\mathrm{CH}]$ for a modern reference. Our approach gives a different (algebraic) proof of the fact that $\sigma_{7}\left(\mathbf{P}^{4}, \mathcal{O}(3)\right)$ is an exceptional case. Another argument, by using syzygies, is in [RS]. B. Reichstein found in [Re] an algorithm to check when a cubic homogeneous polynomial in five variables is the sum of seven cubes, see the Remark 3.4.

The resulting table of the Alexander-Hirschowitz classification is the following

\begin{tabular}{|r|l|l|l|}
\hline & exp. codim & codim & equation \\
\hline $\begin{array}{r}\sigma_{k}\left(\mathbf{P}^{n}, \mathcal{O}(2)\right) \\
2 \leq k \leq n\end{array}$ & $\max \left(\frac{(n+1)(n+2-2 k)}{2}, 0\right)$ & $\left(\begin{array}{c}n-k+2 \\
2\end{array}\right)$ & $(k+1)-$ minors \\
\hline $\begin{array}{r}\sigma_{\frac{1}{2} n(n+3)}\left(\mathbf{P}^{n}, \mathcal{O}(4)\right) \\
n=2,3,4\end{array}$ & 0 & 1 & catalecticant inv. \\
\hline$\sigma_{7}\left(\mathbf{P}^{4}, \mathcal{O}(3)\right)$ & 0 & 1 & see Theorem 1.1 \\
\hline
\end{tabular}

The degree of $\sigma_{k}\left(\mathbf{P}^{n}, \mathcal{O}(2)\right)$ was computed by C. Segre, it is equal to $\prod_{i=0}^{n-k}\left(\begin{array}{c}n+1+i \\ n+1-k-i\end{array}\right) /\left(\begin{array}{c}2 i+1 \\ i\end{array}\right)$. We will use in the proof of Theorem 1.1 the fact that $\sigma_{k-1}\left(\mathbf{P}^{n}, \mathcal{O}(2)\right)$ is the singular locus of $\sigma_{k}\left(\mathbf{P}^{n}, \mathcal{O}(2)\right)$ for $k \leq n$.

A general cubic polynomial in five variables can be expressed as a sum of eight cubes in $\infty^{5}$ ways, parametrized by a Fano 5 -fold of index one (see $[\mathrm{RS}])$. A cubic polynomial in five variables which can be expressed as a sum of seven cubes was called degenerate in [RS], hence what we have found is the locus of degenerate cubics. A degenerate cubic in five variables can be expressed as a sum of seven cubes in $\infty^{1}$ ways, parametrized by $\mathbf{P}^{1}$ (see [RS, 4.2]).

To explain our technique, we consider the Aronhold invariant of plane cubics.

The Aronhold invariant is the degree 4 equation of $\sigma_{3}\left(\mathbf{P}^{2}, \mathcal{O}(3)\right)$, which can be seen as the $S L(3)$-orbit of the Fermat cubic $x_{0}^{3}+x_{1}^{3}+x_{2}^{3}$ (sum of three cubes), see [St, Prop. 4.4.7] or [DK, (5.13.1)].

Let $W$ be a vector space of dimension 3 . In particular $\Gamma^{2,1} W=\operatorname{ad} W$ is self-dual and it has dimension 8 . We get 
TheOREM 1.2. For any $\phi \in S^{3} W$, let $A_{\phi}: \Gamma^{2,1} W \rightarrow \Gamma^{2,1} W$ be the $S L(V)$-invariant contraction operator. Then $A_{\phi}$ is skew-symmetric and the pfaffian Pf $A_{\phi}$ is the equation of $\sigma_{3}(\mathbf{P}(W), \mathcal{O}(3))$, i.e. it is the Aronhold invariant.

The corresponding picture is
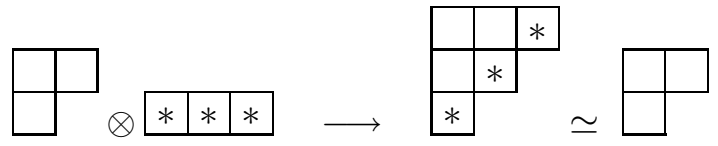

The Aronhold invariant gives the necessary condition to express a cubic homogeneous polynomial in three variables as a sum of three cubes. The explicit expression of the Aronhold invariant is known since the 19th century, but we have not found in the literature its representation as a pfaffian. In the Remark 2.3 we apply this representation to the Scorza map between plane quartics.

In Section 2 we give the proof of Theorem 1.2. This is introductory to Theorem 1.1, which is proved in Section 3. In Section 4 we review, for completeness, some known facts about the catalecticant invariant of quartic hypersurfaces.

We are indebted to S. Sullivant, for his beautiful lectures at Nordfjordeid in 2006 about [SS], where a representation of the Aronhold invariant is found with combinatorial techniques.

\section{§2. The Aronhold invariant as a pfaffian}

Let $e_{0}, e_{1}, e_{2}$ be a basis of $W$ and fix the orientation $\bigwedge^{3} W \simeq K$ given by $e_{0} \wedge e_{1} \wedge e_{2}$. We have End $W=$ ad $W \oplus K$. The $S L(W)$-module ad $W=\Gamma^{2,1}(W)$ consists of the subspace of endomorphisms of $W$ with zero trace. We may interpret the contraction

$$
A_{\phi}: \Gamma^{2,1} W \longrightarrow \Gamma^{2,1} W
$$

as the restriction of a linear map $A_{\phi}^{\prime}$ : End $W \rightarrow$ End $W$, which is defined for $\phi=e_{i_{1}} e_{i_{2}} e_{i_{3}}$ as

$$
A_{e_{i_{1}} e_{i_{2}} e_{i_{3}}}^{\prime}(M)(w)=\sum_{\sigma}\left(M\left(e_{i_{\sigma(1)}}\right) \wedge e_{i_{\sigma(2)}} \wedge w\right) e_{i_{\sigma(3)}}
$$

where $M \in$ End $W, w \in W$ and $\sigma$ covers the symmetric group $\Sigma_{3}$. 
Then $A_{\phi}^{\prime}$ is defined for a general $\phi$ by linearity, and it follows from the definition that it is $S L(V)$-invariant.

The Killing scalar product on End $W$ is defined by $\operatorname{tr}(M \cdot N)$.

Lemma 2.1. (i) $\operatorname{Im}\left(A_{\phi}^{\prime}\right) \subseteq$ ad $W \quad K \subseteq \operatorname{Ker}\left(A_{\phi}^{\prime}\right)$

(ii) $A_{\phi}^{\prime}$ is skew-symmetric.

Proof. (i) follows from

$$
\begin{aligned}
\operatorname{tr}\left[A_{e_{i_{1}} e_{i_{2}} e_{i_{3}}}(M)\right] & =\sum_{s} A_{e_{i_{1}} e_{i_{2}} e_{i_{3}}}(M)\left(e_{s}\right) e_{s}^{\vee} \\
& =\sum_{\sigma}\left(M\left(e_{i_{\sigma(1)}}\right) \wedge e_{i_{\sigma(2)}} \wedge e_{i_{\sigma(3)}}\right)=0
\end{aligned}
$$

The second inclusion is evident. To prove (ii), we have to check that

$$
\operatorname{tr}\left(A_{\phi}(M) \cdot N\right)=-\operatorname{tr}\left(A_{\phi}(N) \cdot M\right)
$$

for $M, N \in$ End $W$. Indeed let $\phi=e_{i_{1}} e_{i_{2}} e_{i_{3}}$. We get

$$
\begin{aligned}
\operatorname{tr}\left(A_{e_{i_{1}} e_{i_{2}} e_{i_{3}}}(M) \cdot N\right) & =\sum_{s} A_{e_{i_{1}} e_{i_{2}} e_{i_{3}}}(M)\left(N\left(e_{s}\right)\right) e_{s}^{\vee} \\
& =\sum_{\sigma} M\left(e_{i_{\sigma(1)}}\right) \wedge e_{i_{\sigma(2)}} \wedge N\left(e_{i_{\sigma(3)}}\right)
\end{aligned}
$$

which is alternating in $M$ and $N$, where we denoted by $e_{i}^{\vee}$ the dual basis.

It follows from Lemma 2.1 that the restriction

$$
A_{\phi \mid \text { ad } W}^{\prime}: \operatorname{ad} W \longrightarrow \operatorname{ad} W
$$

coincides, up to scalar multiple, with the contraction operator $A_{\phi}$ of Theorem 1.2 and it is skew-symmetric.

Lemma 2.2. Let $\phi=w^{3}$ with $w \in W$. Then $\mathrm{rk} A_{\phi}=2$. More precisely

$$
\operatorname{Im} A_{w^{3}}=\{M \in \operatorname{ad} W \mid \operatorname{Im} M \subseteq\langle w\rangle\}
$$

$\operatorname{Ker} A_{w^{3}}=\{M \in \operatorname{ad} W \mid w$ is an eigenvector of $M\}$ 
Proof. The statement follows from the equality

$$
A_{w^{3}}(M)(v)=6(M(w) \wedge w \wedge v) w
$$

As an example, note that $\operatorname{Im} A_{e_{0}^{3}}=\left\langle e_{0} \otimes e_{1}^{\vee}, e_{0} \otimes e_{2}^{\vee}\right\rangle$ and $\operatorname{Ker} A_{e_{0}^{3}}$ is spanned by all the basis monomials, with the exception of $e_{0}^{\vee} \otimes e_{1}$ and $e_{0}^{\vee} \otimes e_{2}$. Due to the $S L(W)$-invariance, this example proves the general case.

Proof of Theorem 1.2. Let $\phi \in \sigma_{3}(\mathbf{P}(W), \mathcal{O}(3))$. By the definition of higher secant variety, $\phi$ is in the closure of elements which can be written as $\phi_{1}+\phi_{2}+\phi_{3}$ with $\phi_{i} \in(\mathbf{P}(W), \mathcal{O}(3))$. From Lemma 2.2 it follows that

$$
\mathrm{rk} A_{\phi} \leq \mathrm{rk} A_{\sum_{i=1}^{3} \phi_{i}}=\mathrm{rk} \sum_{i=1}^{3} A_{\phi_{i}} \leq \sum_{i=1}^{3} \operatorname{rk} A_{\phi_{i}}=2 \cdot 3=6
$$

Hence $\operatorname{Pf}\left(A_{\phi}\right)$ has to vanish on $\sigma_{3}(\mathbf{P}(W), \mathcal{O}(3))$.

Write a cubic polynomial as

$$
\begin{gathered}
\phi=v_{000} x_{0}^{3}+3 v_{001} x_{0}^{2} x_{1}+3 v_{002} x_{0}^{2} x_{2}+3 v_{011} x_{0} x_{1}^{2}+6 v_{012} x_{0} x_{1} x_{2} \\
+3 v_{022} x_{0} x_{2}^{2}+v_{111} x_{1}^{3}+3 v_{112} x_{1}^{2} x_{2}+3 v_{122} x_{1} x_{2}^{2}+v_{222} x_{2}^{3}
\end{gathered}
$$

We order the monomial basis of $\bigwedge^{2} W \otimes W$ with the lexicographical order in the following way:

$$
\begin{aligned}
& \left(w_{0} \wedge w_{1}\right) w_{0},\left(w_{0} \wedge w_{1}\right) w_{1}, \quad\left(w_{0} \wedge w_{1}\right) w_{2}, \\
& \left(w_{0} \wedge w_{2}\right) w_{0},\left(w_{0} \wedge w_{2}\right) w_{1},\left(w_{0} \wedge w_{2}\right) w_{2}, \\
& \left(w_{1} \wedge w_{2}\right) w_{0},\left(w_{1} \wedge w_{2}\right) w_{1},\left(w_{1} \wedge w_{2}\right) w_{2}
\end{aligned}
$$

Call $M_{i}$ for $i=1, \ldots, 9$ this basis. The matrix of $A_{\phi}^{\prime}$, with respect to this basis, has at the entry $(i, j)$ the value $A_{\phi}^{\prime}\left(M_{j}\right)\left(M_{i}\right)$ and it is the following

$$
\left[\begin{array}{rrrrrrrrr}
0 & v_{222} & -v_{122} & 0 & -v_{122} & v_{112} & 0 & v_{022} & -v_{012} \\
-v_{222} & 0 & v_{022} & v_{122} & 0 & -v_{012} & -v_{022} & 0 & v_{002} \\
v_{122} & -v_{022} & 0 & -v_{112} & v_{012} & 0 & v_{012} & -v_{002} & 0 \\
0 & -v_{122} & v_{112} & 0 & v_{112} & -v_{111} & 0 & -v_{012} & v_{011} \\
v_{122} & 0 & -v_{012} & -v_{112} & 0 & v_{011} & v_{012} & 0 & -v_{001} \\
-v_{112} & v_{012} & 0 & v_{111} & -v_{011} & 0 & -v_{011} & v_{001} & 0 \\
0 & v_{022} & -v_{012} & 0 & -v_{012} & v_{011} & 0 & v_{002} & -v_{001} \\
-v_{022} & 0 & v_{002} & v_{012} & 0 & -v_{001} & -v_{002} & 0 & v_{000} \\
v_{012} & -v_{002} & 0 & -v_{011} & v_{001} & 0 & v_{001} & -v_{000} & 0
\end{array}\right]
$$


Deleting one of the columns corresponding to $\left(w_{0} \wedge w_{1}\right) w_{2},\left(w_{0} \wedge w_{2}\right) w_{1}$ or $\left(w_{1} \wedge w_{2}\right) w_{0}$ (respectively the $3 \mathrm{rd}$, the 5 th and the 7 th, indeed their alternating sum gives the trace), and the corresponding row, we get a skewsymmetric matrix of order 8 which is the matrix of $A_{\phi}$. To conclude the proof, it is enough to check that the pfaffian is nonzero. This can be easily checked on the point corresponding to $\phi=x_{0} x_{1} x_{2}$, that is when $v_{012}=1$ and all the other coordinates are equal to zero. This means that any triangle is not in the closure of the Fermat curve. we conclude that $\operatorname{Pf}\left(A_{\phi}\right)$ is the Aronhold invariant. We verified that it coincides, up to a constant, with the expression given in [St, Prop. 4.4.7] or in [DK, (5.13.1)].

The vanishing of the Aronhold invariant gives the necessary and sufficient condition to express a cubic polynomial in three variables as the sum of three cubes.

Remark. $A_{\phi}^{\prime}$ can be thought as a map

$$
A_{\phi}^{\prime}: \bigwedge^{2} W \otimes W \longrightarrow \bigwedge^{2} W^{\vee} \otimes W^{\vee}
$$

For $\phi=w^{3}$ we have the formula

$$
A_{\phi}^{\prime}(\omega \otimes v)\left(\omega^{\prime} \otimes v^{\prime}\right)=(\omega \wedge w) \otimes\left(v \wedge w \wedge v^{\prime}\right) \otimes\left(\omega^{\prime} \wedge w\right)
$$

This is important for the understanding of the next section.

Remark. We have the decomposition

$$
\bigwedge^{2}\left(\Gamma^{2,1} W\right)=S^{3} W \oplus \Gamma^{2,2,2} W \oplus \operatorname{ad} W
$$

and it is a nice exercise to show the behaviour of the three summands. For the first one

$$
S^{3} W \cap\left\{M \in \bigwedge^{2}\left(\Gamma^{2,1} W\right) \mid \operatorname{rk}(M) \leq 2 k\right\}
$$

is the cone over $\sigma_{k}(\mathbf{P}(W), \mathcal{O}(3))$, so that we have found the explicit equations for all the higher secant varieties to $(\mathbf{P}(W), \mathcal{O}(3))$. The secant variety $\sigma_{2}(\mathbf{P}(W), \mathcal{O}(3))$ is the closure of the orbit of plane cubics consisting of three concurrent lines, and its equations are the $6 \times 6$ subpfaffians of $A_{\phi}$. It has degree 15. There is a dual description for $\Gamma^{2,2,2} W$.

For the third summand, we have that

$$
\text { ad } W \subseteq\left\{M \in \bigwedge^{2}\left(\Gamma^{2,1} W\right) \mid \operatorname{rk}(M) \leq 6\right\}
$$


Indeed any $M \in$ ad $W$ induces the skew-symmetric morphism

$$
[M,-]
$$

whose kernel contains $M$. Moreover

$$
\operatorname{ad} W \cap\left\{M \in \bigwedge^{2}\left(\Gamma^{2,1} W\right) \mid \operatorname{rk}(M) \leq 4\right\}
$$

is the 5-dimensional affine cone consisting of endomorphisms $M \in \operatorname{ad} W$ such that their minimal polynomial has degree $\leq 2$.

Remark 2.3. We recall from $[\mathrm{DK}]$ the definition of the Scorza map. Let $A$ be the Aronhold invariant. For any plane quartic $F$ and any point $x \in \mathbf{P}(W)$ we consider the polar cubic $P_{x}(F)$. Then $A\left(P_{x}(F)\right)$ is a quartic in the variable $x$ which we denote by $S(F)$. The rational map $S: \mathbf{P}\left(S^{4} W\right) \rightarrow$ $\mathbf{P}\left(S^{4} W\right)$ is called the Scorza map. Our description of the Aronhold invariant shows that $S(F)$ is defined as the degeneracy locus of a skew-symmetric morphism on $\mathbf{P}(W)$

$$
\mathcal{O}(-2)^{8} \stackrel{f}{\longrightarrow} \mathcal{O}(-1)^{8}
$$

It is easy to check (see [Be]) that Coker $f=E$ is a rank two vector bundle over $S(F)$ such that $c_{1}(E)=K_{S(F)}$. Likely from $E$ it is possible to recover the even theta-characteristic $\theta$ on $S(F)$ defined in [DK, (7.7)]. The natural guess is that

$$
h^{0}(E \otimes(-\theta))>0
$$

for a unique even $\theta$, but we do not know if this is true.

\section{$\S 3 . \quad$ The invariant for cubic polynomials in five variables}

Let now $e_{0}, \ldots, e_{4}$ be a basis of $V$, no confusion will arise with the notations of the previous section. We fix the orientation $\wedge^{5} V \simeq K$ given by $e_{0} \wedge e_{1} \wedge e_{2} \wedge e_{3} \wedge e_{4}$. We construct, for $\phi \in S^{3} V$, the contraction operator

$$
B_{\phi}^{\prime}: \bigwedge^{4} V \otimes \bigwedge^{2} V \longrightarrow \bigwedge^{4} V^{\vee} \otimes \bigwedge^{2} V^{\vee} \simeq \bigwedge^{3} V \otimes V
$$

If $\phi=e_{i_{1}} e_{i_{2}} e_{i_{3}}$, the definition is

$$
\begin{aligned}
& B_{\phi}^{\prime}\left(v_{a} \wedge v_{b} \wedge v_{c} \wedge v_{d}\right) \otimes\left(v_{e} \wedge v_{f}\right) \\
& =\sum_{\sigma}\left(v_{a} \wedge v_{b} \wedge v_{c} \wedge v_{d} \wedge e_{i_{\sigma(1)}}\right) \otimes\left(v_{e} \wedge v_{f} \wedge e_{i_{\sigma(2)}}\right) \otimes e_{i_{\sigma(3)}}
\end{aligned}
$$


where $\sigma$ covers the symmetric group $\Sigma_{3}$ and we extend this definition, to a general $\phi$, by linearity.

We may interpret $B_{\phi}^{\prime}$ as a morphism

$$
B_{\phi}^{\prime}: \operatorname{Hom}\left(V, \bigwedge^{2} V\right) \longrightarrow \operatorname{Hom}\left(\bigwedge^{2} V, V\right)
$$

If $\phi=e_{i_{1}} e_{i_{2}} e_{i_{3}}$ and $M \in H o m\left(V, \bigwedge^{2} V\right)$ we have

$$
B_{e_{i_{1}} e_{i_{2}} e_{i_{3}}}^{\prime}(M)\left(v_{1} \wedge v_{2}\right)=\sum_{\sigma}\left(M\left(e_{i_{\sigma(1)}}\right) \wedge e_{i_{\sigma(2)}} \wedge v_{1} \wedge v_{2}\right) e_{i_{\sigma(3)}}
$$

We have a $S L(V)$-decomposition

$$
\bigwedge^{4} V \otimes \bigwedge^{2} V=\Gamma^{2,2,1,1} V \oplus V
$$

Consider the contraction $c: \bigwedge^{4} V \otimes \bigwedge^{2} V \rightarrow V$ defined by

$$
c\left(\omega \otimes\left(v_{i} \wedge v_{j}\right)\right)=\left(\omega \wedge v_{i}\right) v_{j}-\left(\omega \wedge v_{j}\right) v_{i}
$$

Then the subspace $\Gamma^{2,2,1,1} V$ can be identified with

$$
\left\{M \in \bigwedge^{4} V \otimes \bigwedge^{2} V \mid c(M)=0\right\}
$$

or with

$$
\left\{M \in \operatorname{Hom}\left(V, \bigwedge^{2} V\right) \mid \sum e_{i}^{\vee} M\left(e_{i}\right)=0\right\}
$$

The subspace $V \subset \operatorname{Hom}\left(V, \wedge^{2} V\right)$ can be identified with $\{v \wedge-\mid v \in V\}$. At the same time we have a $S L(V)$-decomposition

$$
V \otimes \bigwedge^{3} V=\Gamma^{2,1,1} V \oplus \bigwedge^{4} V
$$

and the obvious contraction $d: V \otimes \bigwedge^{3} V \rightarrow \bigwedge^{4} V$. The subspace $\Gamma^{2,1,1} V$ can be identified with

$$
\left\{N \in V \otimes \bigwedge^{3} V \mid d(N)=0\right\}
$$

Lemma 3.1. (i) $\operatorname{Im}\left(B_{\phi}^{\prime}\right) \subseteq \Gamma^{2,1,1} V \quad V \subseteq \operatorname{Ker}\left(B_{\phi}^{\prime}\right)$

(ii) $B_{\phi}^{\prime}$ is symmetric. 
Proof. The statement (i) follows from the formula

$$
\begin{aligned}
& d\left(B_{e_{i_{1}} e_{i_{2}} e_{i_{3}}}^{\prime}\left(v_{a} \wedge v_{b} \wedge v_{c} \wedge v_{d}\right) \otimes\left(v_{e} \wedge v_{f}\right)\right) \\
& =\sum_{\sigma}\left(v_{a} \wedge v_{b} \wedge v_{c} \wedge v_{d} \wedge e_{i_{\sigma(1)}}\right) \otimes\left(v_{e} \wedge v_{f} \wedge e_{i_{\sigma(2)}} \wedge e_{i_{\sigma(3)}}\right)=0
\end{aligned}
$$

In order to prove the second inclusion, for any $v \in V$ consider the induced morphism $M_{v}(w)=v \wedge w$. We get

$$
B_{e_{i_{1}} e_{i_{2}} e_{i_{3}}}^{\prime}\left(M_{v}\right)\left(v_{1} \wedge v_{2}\right)=\sum_{\sigma}\left(v \wedge e_{i_{\sigma(1)}} \wedge e_{i_{\sigma(2)}} \wedge v_{1} \wedge v_{2}\right) e_{i_{\sigma(3)}}=0
$$

In order to prove (ii) we may assume $\phi=v^{3}$.

We need to prove that

$$
B_{v^{3}}^{\prime}(\omega \otimes \xi)\left(\omega^{\prime} \otimes \xi^{\prime}\right)=B_{v^{3}}^{\prime}\left(\omega^{\prime} \otimes \xi^{\prime}\right)(\omega \otimes \xi)
$$

for every $\omega, \omega^{\prime} \in \bigwedge^{4} V$ and $\xi, \xi^{\prime} \in \bigwedge^{2} V$. Indeed

$$
B_{v^{3}}^{\prime}(\omega \otimes \xi)\left(\omega^{\prime} \otimes \xi^{\prime}\right)=(\omega \wedge v) \otimes\left(\xi \wedge v \wedge \xi^{\prime}\right) \otimes\left(v \wedge \omega^{\prime}\right)
$$

which is symmetric in the pair $(\omega, \xi)$.

It follows from Lemma 3.1 that the restriction $B_{\phi \mid \Gamma^{2,2,1,1}}^{\prime}: \Gamma^{2,2,1,1} \rightarrow$ $\Gamma^{2,1,1} V$ coincides, up to scalar multiple, with the contraction $B_{\phi}$ of the Theorem 1.1 and it is symmetric. Note that

$$
\operatorname{Ker}\left(B_{\phi}\right)=\operatorname{Ker}\left(B_{\phi}^{\prime}\right) / V \quad \operatorname{Im}\left(B_{\phi}\right)=\operatorname{Im}\left(B_{\phi}^{\prime}\right)
$$

Lemma 3.2. Let $\phi=v^{3}$ with $v \in V$. Then $\mathrm{rk} B_{\phi}=6$. More precisely

$$
\begin{aligned}
\operatorname{Im} B_{v^{3}}=\left\{N \in \operatorname{Hom}\left(\bigwedge^{2} V, V\right) \mid \sum e_{i}^{\vee} N\left(e_{i} \wedge v\right)=0,\right. & \\
& \forall v \in V, \quad \operatorname{Im}(N) \subseteq\langle v\rangle\}
\end{aligned}
$$

$\operatorname{Ker} B_{v^{3}}=\left\{M \in \operatorname{Hom}\left(V, \bigwedge^{2} V\right) \mid \sum e_{i}^{\vee} M\left(e_{i}\right)=0, M(v) \subseteq v \wedge V\right\}$

Proof. The statement follows from the equality

$$
B_{v^{3}}(M)\left(v_{1} \wedge v_{2}\right)=6\left(M(v) \wedge v \wedge v_{1} \wedge v_{2}\right) v
$$

As an example, a basis of $\operatorname{Im} B_{e_{0}^{3}}$ is given by $e_{0} \otimes\left(e_{i}^{\vee} \wedge e_{j}^{\vee}\right)$ for $1 \leq i<$ $j \leq 4$ and a basis of $\operatorname{Ker} B_{e_{0}^{3}}$ is given by all the basis monomials with the exceptions of $e_{0}^{\vee} \otimes\left(e_{i} \wedge e_{j}\right)$ for $1 \leq i<j \leq 4$. Due to the $S L(V)$-invariance, this example proves the general case. 
We write $\phi \in S^{3} V$ as $\phi=v_{000} x_{0}^{3}+3 v_{001} x_{0}^{2} x_{1}+\cdots+v_{444} x_{4}^{3}$.

LEMMA 3.3. Every $S L(V)$-invariant homogeneous polynomial of degree 15 on $S^{3} V$ which contains the monomial

$$
v_{000}^{2} v_{012}^{3} v_{111} v_{223}^{3} v_{334}^{3} v_{144}^{3}
$$

is irreducible.

Proof. Let $t_{0}, \ldots, t_{4}$ be the canonical basis of $\mathbb{Z}^{5}$. We denote by $t_{i}+$ $t_{j}+t_{k}$ the weight of the monomial $v_{i j k}$, according to [St]. For example the weight of $v_{000}$ is $(3,0,0,0,0)$. We denote the first component of the weight as the $x_{0}$-weight, the second component as the $x_{1}$-weight, and so on. We recall that every $S L(V)$-invariant polynomial is isobaric, precisely every monomial of a $S L(V)$-invariant polynomial of degree $5 k$ has weight $(3 k, 3 k, 3 k, 3 k, 3 k)$ (see [St, (4.4.14)]), this follows from the invariance with respect to the diagonal torus. We claim that there is no isobaric monomial of weight $(6,6,6,6,6)$ and degree 10 with variables among $v_{000}, v_{012}, v_{111}, v_{223}$, $v_{334}, v_{144}$. We divide into the following cases, by looking at the possibilities for the $x_{0}$-weight:

i) The monomial contains $v_{000}^{2}$ and does not contain $v_{012}$. By looking at the $x_{2}$-weight, the monomial has to contain $v_{223}^{3}$, which gives contribution 3 to the $x_{3}$-weight. This gives a contradiction, because from $v_{334}$ the possible values for the $x_{3}$-weight are even, and we never make 6.

ii) The monomial contains $v_{000} v_{012}^{3}$ and not higher powers. This monomial gives contribution 3 to the $x_{2}$-weight. From $v_{223}$ the possible values for the $x_{2}$-weight are even, and we never make 6 , again.

iii) The monomial contains $v_{012}^{6}$ and does not contain $v_{000}$. This monomial gives contribution 6 to the $x_{0}$-weight, and the same contribution is given to the $x_{1}$-weight and to the $x_{2}$-weight. Hence the only other possible monomial that we are allowed to use is $v_{334}$, which gives a $x_{3}$ weight doubled with respect to the $x_{4}$-weight, which is a contradiction.

This contradiction proves our claim. Nevertheless, if our polynomial is reducible, also its factors have to be homogeneous and $S L(V)$-invariant, and the monomial in the statement should split into two factors of degree 5 and 10 , against the claim. 
Proof of Theorem 1.1. Let $\phi \in \sigma_{7}(\mathbf{P}(V), \mathcal{O}(3))$. By the definition of higher secant variety, $\phi$ is in the closure of elements which can be written as $\sum_{i=1}^{7} \phi_{i}$ with $\phi_{i} \in(\mathbf{P}(V), \mathcal{O}(3))$. From Lemma 3.2 it follows that

$$
\operatorname{rk} B_{\phi} \leq \operatorname{rk} B_{\sum_{i=1}^{7} \phi_{i}}=\operatorname{rk} \sum_{i=1}^{7} B_{\phi_{i}} \leq \sum_{i=1}^{7} \operatorname{rk} B_{\phi_{i}}=6 \cdot 7=42
$$

Hence $\operatorname{det}\left(B_{\phi}\right)$ has to vanish on $\sigma_{7}(\mathbf{P}(V), \mathcal{O}(3))$.

We order the monomial basis of $S^{3} V$ with the lexicographical ordered induced by $x_{0}<x_{1}<x_{2}<x_{3}<x_{4}$. We order also the basis of $\bigwedge^{2} V \otimes \bigwedge^{4} V$ with the lexicographical order. There are 50 terms, beginning with

$$
\left(e_{0} \wedge e_{1}\right) \otimes\left(e_{0} \wedge e_{1} \wedge e_{2} \wedge e_{3}\right),\left(e_{0} \wedge e_{1}\right) \otimes\left(e_{0} \wedge e_{1} \wedge e_{2} \wedge e_{4}\right), \ldots
$$

and ending with

$$
\ldots,\left(e_{3} \wedge e_{4}\right) \otimes\left(e_{1} \wedge e_{2} \wedge e_{3} \wedge e_{4}\right)
$$

These 50 terms are divided into 10 blocks, depending on the first factor $e_{s} \wedge e_{t}$. The matrix of $B_{\phi}^{\prime}$, with respect to this basis, is a $50 \times 50$ symmetric matrix with linear monomial entries from $v_{i j k}$.

We describe this matrix in block form. For $i=0, \ldots, 4$ let $A_{i}$ be the $5 \times 5$ symmetric matrix which at the entry $(5-s, 5-t)$ has $(-1)^{s+t} v_{i s t}$, corresponding to the monomial $x_{i} x_{s} x_{t}$. For example

$$
A_{4}=\left[\begin{array}{rrrrr}
v_{444} & -v_{344} & v_{244} & -v_{144} & v_{044} \\
-v_{344} & v_{334} & -v_{234} & v_{134} & -v_{034} \\
v_{244} & -v_{234} & v_{224} & -v_{124} & v_{024} \\
-v_{144} & v_{134} & -v_{124} & v_{114} & -v_{014} \\
v_{044} & -v_{034} & v_{024} & -v_{014} & v_{004}
\end{array}\right]
$$

Then the matrix of $B_{\phi}^{\prime}$ has the following block form

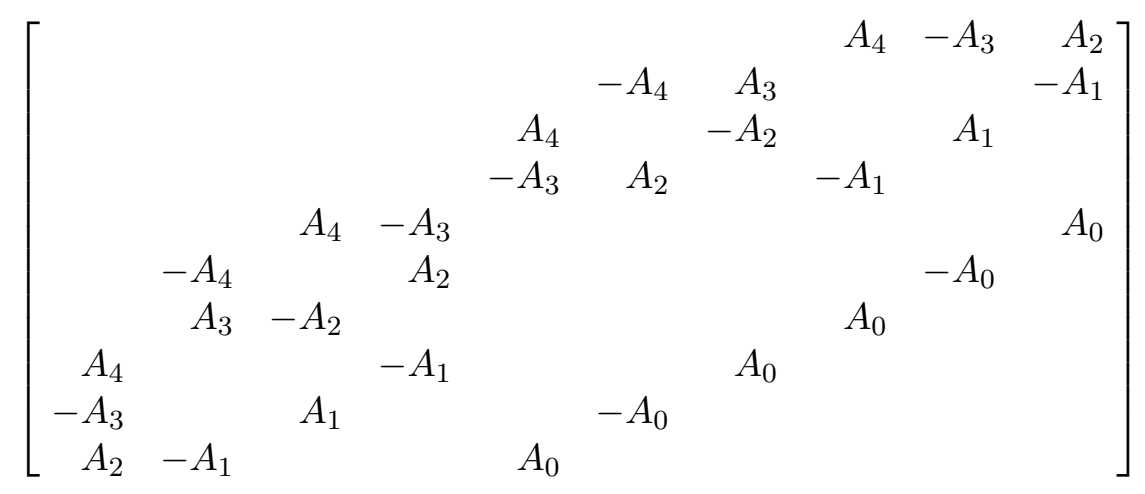


Among the 50 basis elements, there are 30 tensors $\left(e_{s} \wedge e_{t}\right) \otimes\left(e_{i} \wedge e_{j} \wedge\right.$ $\left.e_{k} \wedge e_{l}\right)$ such that $\{s, t\} \subseteq\{i, j, k, l\}$. The other 20 elements are divided into 5 groups, depending on the single index $\{s, t\} \cap\{i, j, k, l\}$. The contraction $c$ maps the first group of 30 elements into 30 independent elements of $\Gamma^{2,2,1,1} V$, and each group of 4 elements has the image through $c$ of dimension 3 in $\Gamma^{2,2,1,1} V$, indeed the images of the 4 elements satisfy a linear relation with \pm 1 coefficients.

It follows that the matrix of $B_{\phi}$ can be obtained from the matrix of $B_{\phi}^{\prime}$ by deleting five rows, one for each of the above groups, and the corresponding five columns. We can delete, for example, the columns and the rows corresponding to

$$
\begin{aligned}
& \left(e_{0} \wedge e_{1}\right) \otimes\left(e_{1} \wedge e_{2} \wedge e_{3} \wedge e_{4}\right),\left(e_{0} \wedge e_{2}\right) \otimes\left(e_{1} \wedge e_{2} \wedge e_{3} \wedge e_{4}\right), \\
& \left(e_{0} \wedge e_{3}\right) \otimes\left(e_{1} \wedge e_{2} \wedge e_{3} \wedge e_{4}\right),\left(e_{0} \wedge e_{4}\right) \otimes\left(e_{0} \wedge e_{1} \wedge e_{2} \wedge e_{3}\right), \\
& \left(e_{0} \wedge e_{4}\right) \otimes\left(e_{1} \wedge e_{2} \wedge e_{3} \wedge e_{4}\right)
\end{aligned}
$$

which have respectively number $5,10,15,16,20$. Note that in the resulting matrix for $B_{\phi}$, all entries are monomials in $v_{i j k}$ with coefficient \pm 1 .

In order to show that for general $\phi$ the morphism $B_{\phi}$ is invertible, the simplest way is to look at the monomial $\left(v_{001} v_{022} v_{113} v_{244} v_{334}\right)^{9}$ which appears with nonzero coefficient in the expression of $\operatorname{det} B_{\phi}$. We prefer instead to use the monomial appearing in the statement of Lemma 3.3, which allows to prove the stronger statement that $\operatorname{det} B_{\phi}$ is the cube of an irreducible polynomial. Indeed, by substituting 0 to all the variables different from $v_{000}, v_{012}, v_{111}, v_{223}, v_{334}, v_{144}$, we get by an explicit computation that the determinant is equal to

$$
-2\left(v_{000}^{2} v_{012}^{3} v_{111} v_{223}^{3} v_{334}^{3} v_{144}^{3}\right)^{3}
$$

Hence for general $\phi$ we have $\mathrm{rk} B_{\phi}=45$. Note that this gives an alternative proof of the fact that $\sigma_{7}(\mathbf{P}(V), \mathcal{O}(3))$ has codimension bigger than zero, and it has to appear in the Alexander-Hirschowitz classification. It follows that on the points of $\sigma_{7}(\mathbf{P}(V), \mathcal{O}(3))$ the rank of $\mathrm{rk} B_{\phi}$ drops at least by three, so that $\sigma_{7}(\mathbf{P}(V), \mathcal{O}(3))$ is contained in the singular locus of $\operatorname{det} B_{\phi}$, and in particular $\operatorname{det} B_{\phi}$ has to vanish with multiplicity $\geq 3$ on $\sigma_{7}(\mathbf{P}(V), \mathcal{O}(3))$. It is known that $\sigma_{7}(\mathbf{P}(V), \mathcal{O}(3))$ is a hypersurface (see $[\mathrm{CH}]$ ), hence its equation $P$ has to be a factor of multiplicity $\geq 3$ of $\operatorname{det} B_{\phi}$. Since every $S L(V)$-invariant polynomial has degree $5 k$, the possible values for the degree of $P$ are 5, 10 or 15 . Look at the monomials in $P$ containing some among 
the variables $v_{000}, v_{012}, v_{111}, v_{223}, v_{334}, v_{144}$, these monomials have to exist, due to the explicit computation performed before. If the degree of $P$ is $\leq 10$, then there exists a $S L(V)$-invariant polynomial of degre 10 with a monomial containing the above variables, but this contradicts the claim proved along the proof of the Lemma 3.3. It follows that $\operatorname{deg} P=\operatorname{deg} \sigma_{7}(\mathbf{P}(V), \mathcal{O}(3))=$ 15 and $P^{3}$ divides $\operatorname{det} B_{\phi}$, looking again at our explicit computation we see that we can arrange the scalar multiples in order that $P$ is defined over the rational numbers (as all the $S L(V)$-invariants) and the equation $2 P(\phi)^{3}=\operatorname{det} B_{\phi}$ holds. The Lemma 3.3 shows that $P$ is irreducible.

Remark 3.4. The results obtained by Reichstein with his algorithm developed in [Re] can be verified with the Theorem 1.1. For example when $w$ is like in the Example 1 at page 48 of [Re], a computer check shows that $\operatorname{rk}\left(B_{w}\right)=42$, confirming that $w \in \sigma_{7}(\mathbf{P}(V), \mathcal{O}(3))$, while when $w$ is like in the Example 2 at page 57 of [Re] then $\operatorname{rk}\left(B_{w}\right)=45$, so that $w \notin \sigma_{7}(\mathbf{P}(V), \mathcal{O}(3))$.

The simplest example of a cubic which is not the sum of seven cubes is probably

$$
\phi=x_{0}^{2} x_{1}+x_{0} x_{2}^{2}+x_{1}^{2} x_{3}+x_{2} x_{4}^{2}+x_{3}^{2} x_{4}
$$

where $\operatorname{det}\left(B_{\phi}\right)=-2$, which can be checked even without a computer, but with a good amount of patience. The polynomial $\phi$ defines a smooth cubic 3-fold.

\section{§4. The catalecticant invariant for Clebsch quartics}

Let $U$ be any vector space of dimension $n+1$.

Every quartic $f \in S^{4} U$ induces the contraction $C_{f}: S^{2} U^{\vee} \rightarrow S^{2} U$. Clebsch realized in 1861 that if $f \in\left(\mathbf{P}^{n}, \mathcal{O}(4)\right)$ then $\operatorname{rk} A_{f}=1$. Indeed, with the notations of the previous sections,

$$
C_{v^{4}}\left(u_{1} u_{2}\right)=24 u_{1}(v) u_{2}(v) v^{2}
$$

is always a scalar multiple of $v^{2}$. Clebsch worked in the case $n=2$ but the same result holds for every $n$. If $f \in \sigma_{k}\left(\mathbf{P}^{n}, \mathcal{O}(4)\right)$, we get that $C_{f}$ is the limit of a sum of $k$ matrices of rank one, then $\operatorname{rk} C_{f} \leq k$. The quartic $f$ is called a Clebsch quartic if and only if $\operatorname{det} C_{f}=0$, and this equation gives the catalecticant invariant (see $[\mathrm{IK}]$ or $[\mathrm{DK}]$ ). A matrix description is the following. Let $D_{i}$ for $i=1, \ldots,\left(\begin{array}{c}n+2 \\ 2\end{array}\right)$ be a basis of differential operators of second order on $U$. Then $\operatorname{det}\left(D_{i} D_{j} f\right)$ is the catalecticant invariant. 
The picture in terms of Young diagrams for $n=2$ is
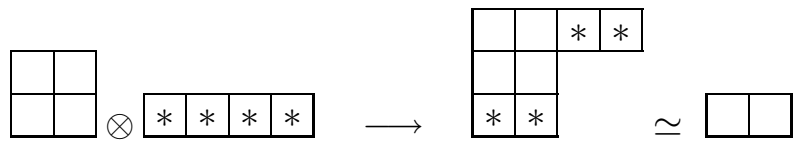

If $n=2$, we write

$f=f_{0000} x_{0}^{4}+4 f_{0001} x_{0}^{3} x_{1}+6 f_{0011} x_{0}^{2} x_{1}^{2}+\cdots+12 f_{0012} x_{0}^{2} x_{1} x_{2}+\cdots+f_{2222} x_{2}^{4}$

Then the well known expression for the degree 6 equation of $\sigma_{5}\left(\mathbf{P}^{2}\right.$, $\mathcal{O}(4)$ ) is the following (we choosed the basis $\partial_{00}, \partial_{01}, \partial_{11}, \partial_{02}, \partial_{12}, \partial_{22}$ )

$$
\operatorname{det}\left[\begin{array}{cccccc}
f_{0000} & f_{0001} & f_{0011} & f_{0002} & f_{0012} & f_{0022} \\
f_{0001} & f_{0011} & f_{0111} & f_{0012} & f_{0112} & f_{0122} \\
f_{0011} & f_{0111} & f_{1111} & f_{0112} & f_{1112} & f_{1122} \\
f_{0002} & f_{0012} & f_{0112} & f_{0022} & f_{0122} & f_{0222} \\
f_{0012} & f_{0112} & f_{1112} & f_{0122} & f_{1122} & f_{1222} \\
f_{0022} & f_{0122} & f_{1122} & f_{0222} & f_{1222} & f_{2222}
\end{array}\right]=0
$$

The above equation gives the necessary condition to express a quartic homogeneous polynomial in 3 variables as the sum of 5 fourth powers. Mukai proves in $[\mathrm{Mu}]$ that a general plane quartic is a sum of 6 fourth powers in $\infty^{3}$ ways, parametrized by the Fano 3 -fold $V_{22}$.

The Clebsch quartics give a hypersurface of degree $\left(\begin{array}{c}n+2 \\ 2\end{array}\right)$ in the space of all quartics.

It follows that this hypersurface contains the variety of $k$-secants to $\left(\mathbf{P}^{n}, \mathcal{O}(4)\right)$ for $k=\left[\left(\begin{array}{c}n+2 \\ 2\end{array}\right)-1\right]=n(n+3) / 2$, and it is equal to this secant variety for $1 \leq n \leq 4$, which turns out to be defective for $2 \leq n \leq 4$. Indeed it is a hypersurface while it is expected that it fills the ambient space. This explains why this example appears in the Alexander-Hirschowitz classification.

Added in proof: F. Schreyer communicated to us that $B_{\phi}$ of the Theorem 1.1 appears also in the apolar ring of $\phi$.

\section{REFERENCES}

[AH] J. Alexander and A. Hirschowitz, Polynomial interpolation in several variables, J. Alg. Geom., 4 (1995), no. 2, 201-222. 
[Be] A. Beauville, Determinantal hypersurfaces, Michigan Math. J., 48 (2000), 39-64.

[BO] M. C. Brambilla and G. Ottaviani, On the Alexander-Hirschowitz Theorem, J. of Pure and Applied Algebra, 212 (2008), 1229-1251.

[CH] C. Ciliberto and A. Hirschowitz, Hypercubiques de $P^{4}$ avec sept points singuliers génériques, C. R. Acad. Sci. Paris Sér. I Math., 313 (1991), no. 3, 135-137.

[DK] I. Dolgachev and V. Kanev, Polar covariants of plane cubics and quartics, Adv. Math., 98 (1993), no. 2, 216-301.

[FH] W. Fulton and J. Harris, Representation theory, Graduate Texts in Math. 129, Springer-Verlag, New York, 1991.

[IK] A. Iarrobino and V. Kanev, Power sums, Gorenstein algebras, and determinantal loci, Lecture Notes in Mathematics 1721, Springer, 1999.

[Mu] S. Mukai, Fano 3-folds, LMS Lecture Notes Series 179, Cambridge, 1992.

[RS] K. Ranestad and F. Schreyer, Varieties of sums of powers, J. Reine Angew. Math., 525 (2000), 147-181.

[Re] B. Reichstein, On Waring's problem for cubic forms, Linear Algebra Appl., 160 (1992), 1-61.

[St] B. Sturmfels, Algorithms in invariant theory, Springer, New York, 1993.

[SS] B. Sturmfels and S. Sullivant, Combinatorial secant varieties, Pure Appl. Math. Q., 2 (2006), no. 3, 867-891.

Dipartimento di Matematica U. Dini

Università di Firenze

viale Morgagni 67/A, 50134 Firenze

Italy

ottavian@math.unifi.it 